\title{
Interferometric data reduction with AMBER/VLTI. Principle, estimators, and illustration ${ }^{\star}$
}

E. Tatulli ${ }^{1,2}$, F. Millour ${ }^{1,3}$, A. Chelli ${ }^{1}$, G. Duvert ${ }^{1}$, B. Acke ${ }^{1,14}$, O. Hernandez Utrera ${ }^{1}$, K.-H. Hofmann ${ }^{4}$, S. Kraus ${ }^{4}$, F. Malbet ${ }^{1}$, P. Mège ${ }^{1}$, R.G. Petrov ${ }^{3}$, M. Vannier ${ }^{3,6,13}$, G. Zins $^{1}$, P. Antonelli ${ }^{5}$, U. Beckmann ${ }^{4}$, Y. Bresson ${ }^{5}$, M. Dugué ${ }^{5}$, S. Gennari ${ }^{2}$, L. Glück ${ }^{1}$, P. Kern ${ }^{1}$, S. Lagarde ${ }^{5}$, E. Le Coarer ${ }^{1}$, F. Lisi ${ }^{2}$, K. Perraut ${ }^{1}$, P. Puget ${ }^{1}$, F. Rantakyrö ${ }^{6}$, S. Robbe-Dubois ${ }^{3}$, A. Roussel ${ }^{5}$, G. Weigelt ${ }^{4}$, M. Accardo ${ }^{2}$, K. Agabi ${ }^{3}$, E. Altariba ${ }^{1}$, B. Arezki ${ }^{1}$, E. Aristidi ${ }^{3}$, C. Baffa ${ }^{2}$, J. Behrend ${ }^{4}$, T. Blöcker ${ }^{4}$, S. Bonhomme ${ }^{5}$, S. Busoni ${ }^{2}$, F. Cassaing ${ }^{7}$, J.-M. Clausse ${ }^{5}$, J. Colin ${ }^{5}$, C. Connot ${ }^{4}$, A. Delboulbé ${ }^{1}$, A. Domiciano de Souza ${ }^{3,5}$, T. Driebe ${ }^{4}$, P. Feautrier ${ }^{1}$, D. Ferruzzi ${ }^{2}$, T. Forveille ${ }^{1}$, E. Fossat ${ }^{3}$, R. Foy $^{8}$, D. Fraix-Burnet ${ }^{1}$, A. Gallardo ${ }^{1}$, E. Giani ${ }^{2}$, C. Gil $^{1,15}$, A. Glentzlin ${ }^{5}$, M. Heiden ${ }^{4}$, M. Heininger $^{4}$, D. Kamm ${ }^{5}$, M. Kiekebusch ${ }^{6}$, D. Le Contel ${ }^{5}$, J.-M. Le Contel ${ }^{5}$, T. Lesourd ${ }^{9}$, B. Lopez ${ }^{5}$, M. Lopez ${ }^{9}$, Y. Magnard ${ }^{1}$, A. Marconi ${ }^{2}$, G. Mars ${ }^{5}$, G. Martinot-Lagarde ${ }^{5,9}$, P. Mathias ${ }^{5}$, J.-L. Monin ${ }^{1}$, D. Mouillet ${ }^{1,16}$, D. Mourard ${ }^{5}$, E. Nussbaum ${ }^{4}$, K. Ohnaka ${ }^{4}$, J. Pacheco ${ }^{5}$, C. Perrier ${ }^{1}$, Y. Rabbia ${ }^{5}$, S. Rebattu ${ }^{5}$, F. Reynaud ${ }^{10}$, A. Richichi ${ }^{11}$, A. Robini ${ }^{3}$, M. Sacchettini ${ }^{1}$, D. Schertl ${ }^{4}$, M. Schöller ${ }^{6}$, W. Solscheid ${ }^{4}$, A. Spang ${ }^{5}$, P. Stee ${ }^{5}$, P. Stefanini ${ }^{2}$, M. Tallon ${ }^{8}$, I. Tallon-Bosc ${ }^{8}$, D. Tasso ${ }^{5}$, L. Testi ${ }^{2}$, F. Vakili ${ }^{3}$, O. von der Lühe ${ }^{12}$, J.-C. Valtier ${ }^{5}$, and N. Ventura ${ }^{1}$

(Affiliations can be found after the references)

Received 2 January 2006 / Accepted 1 March 2006

\section{ABSTRACT}

Aims. In this paper, we present an innovative data reduction method for single-mode interferometry. It has been specifically developed for the AMBER instrument, the three-beam combiner of the Very Large Telescope Interferometer, but it can be derived for any single-mode interferometer Methods. The algorithm is based on a direct modelling of the fringes in the detector plane. As such, it requires a preliminary calibration of the instrument in order to obtain the calibration matrix that builds the linear relationship between the interferogram and the interferometric observable, which is the complex visibility. Once the calibration procedure has been performed, the signal processing appears to be a classical least-square determination of a linear inverse problem. From the estimated complex visibility, we derive the squared visibility, the closure phase, and the spectral differential phase.

Results. The data reduction procedures have been gathered into the so-called amdlib software, now available for the community, and are presented in this paper. Furthermore, each step in this original algorithm is illustrated and discussed from various on-sky observations conducted with the VLTI, with a focus on the control of the data quality and the effective execution of the data reduction procedures. We point out the present limited performances of the instrument due to VLTI instrumental vibrations which are difficult to calibrate.

Key words. technique: interferometric - methods: data analysis - instrumentation: interferometers

\section{Introduction}

AMBER is the first-generation near-infrared three-way beam combiner (Petrov et al. 2007) of the Very Large Telescope Interferometer (VLTI). This instrument simultaneously provides spectrally dispersed visibility for three baselines and a closure phase at three different spectral resolutions. AMBER has been designed to investigate the milli-arcsec surrounding of astrophysical sources like young and evolved stars or active galactic nuclei, and to possibly detect exoplanet signal. The main new feature of this instrument compared to other interferometric instruments is the simultaneous use of modal filters (optical fibers) and a dispersed fringe combiner using spatial coding. The AMBER team has therefore carefully investigated a data processing strategy for this instrument and is providing a new type of data reduction method.

Given the astonishingly quick evolution of ground based optical interferometers in only two decades, in terms of

$\star$ Based on observations collected at the European Southern Observatory, Paranal, Chile. baseline lengths and number of recombined telescopes, the interest of using the practical characteristics of single-mode fibers to carry and recombine the light, as first proposed by Connes et al. (1987) with his conceptual FLOAT interferometer, is now well established. Furthermore, in the light of the FLUOR experiment on the IOTA interferometer, which demonstrated the "on-sky" feasibility of such interferometers for the first time, Coudé Du Foresto et al. (1997) showed that making use of single mode waveguides could also increase the performances of optical interferometry, thanks to their remarkable properties of spatial filtering, which change the phase fluctuations of the atmospheric turbulent wavefront into intensity fluctuations. Indeed, by monitoring these fluctuations in real time thanks to dedicated photometric outputs and by performing instantaneous photometric calibration, he experimentally proved that single-mode interferometry could achieve visibility measurements with precisions of $1 \%$ or lower. Achievement of such performance level has since been confirmed with the IONIC integrated optic beam combiner set up on the same interferometer (LeBouquin et al. 2004). 

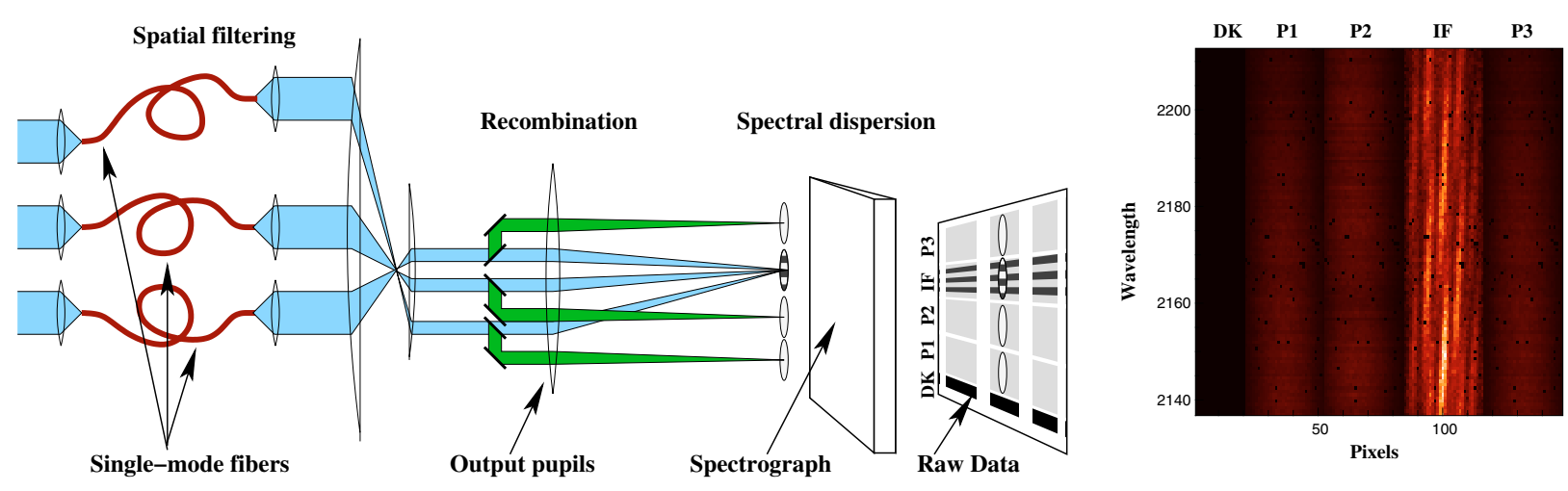

Fig. 1. Left panel: Sketch of the AMBER instrument. The light enters the instrument from the left and is propagating from left to right until the raw data are recorded on the detector. Further details are given in the text. Right panel: AMBER reconstituted image from the raw data recorded during the 3-telescope observation of the calibrator HD135382 in February 2005, in the medium spectral resolution mode. DK corresponds to a dark region, $\mathrm{Pk}$ are the vertically dispersed spectra obtained from each telescope, and IF is the spectrally dispersed interferogram.

Surprisingly, the effect of single-mode waveguides on the interferometric signal has only been studied recently from a theoretical point of view. Ruilier et al. (1997) used numerical simulations in the presence of partial correction by adaptive optics to show that spatial filtering provided a gain on the visibility signal to noise ratio. However his study was limited to the case of a point source. The case of sources with a given spatial extent was first theoretically addressed by Dyer \& Christensen (1999) from a geometrical point of view. They proved that the visibility obtained from single-mode interferometry was biased, the object being multiplied by the antenna lobe (the point spread function of one single telescope) exactly as it happens in radio interferometry (Guilloteau 2001). An equivalent geometrical bias was also characterized for the closure phase (Longueteau et al. 2002). Then Guyon (2002) noticed in his simulations that took the presence of atmospheric turbulence into account, that interferometric observations of extended objects (resolved by one single telescope) could not be completely corrected for atmospheric perturbations, therefore lowering the performances of single-mode interferometry. Finally, by thoroughly describing the propagation of the electric field through single-mode waveguides in the general case of partial correction by adaptive optics and for a source with a given spatial extent, Mège et al. (2003) unified previous studies and introduces the concept of modal visibility, which in the general case does not equal the source visibility $V_{\mathrm{o}}$ and exhibits a jointly geometrical and atmospheric bias. Nevertheless they also show that for compact sources, i.e. smaller than one Airy disk, the mutual coherence factor $\mu$ could be written in the form of a simple product $\mu=T_{i} T_{a} V_{\mathrm{o}}$ where $T_{i}$ and $T_{a}$ are, respectively, the instrumental and the atmospheric transfer functions that can be calibrated. Recently, Tatulli et al. (2004) deduced from an analytical approach that in the specific case of compact objects, the benefit of single-mode waveguides is substantial, not only in terms of the signal-to-noise ratio of the visibility but also of the robustness of the estimator.

Hence, following the path opened by the FLUOR experiment, the AMBER instrument - the three-beam combiner of the VLTI (Petrov et al. 2007) - makes use of the filtering properties of single-mode fibers. However, in contrast to FLUOR, PTI (Colavita 1999a) or VINCI on the VLTI (Kervella et al. 2003), where the fringes are coded temporally with a movable piezzo-electric mirror, the interference pattern is scanned spatially thanks to separated output pupils, the separation fixing the spatial coding frequency of the fringes, as in the case of the GI2T interferometer (Mourard et al. 2000). Thus, if data reduction methods have already been proposed for single-mode interferometers using temporal coding (Colavita 1999b; Kervella et al. 2004), this paper is the first to present a signal-processing algorithm dedicated to single-mode interferometry with spatial beam recombination. Moreover, in the case of AMBER, the configuration of the output pupils, i.e. the spatial coding frequency, imposes a partial overlap of the in the three telescopes case interferometric peaks in the Fourier plane. As a consequence, data reduction based on the classical estimators in the Fourier plane (Roddier \& Lena 1984; Mourard et al. 1994) cannot be performed. The AMBER data reduction procedure is based on a direct analysis in the detector plane, a principle that is an optimization of the "ABCD" estimator as derived in Colavita (1999b). The specificity of the AMBER coding and its subsequent estimation of the observables arises from the desire to characterize and to make use of the linear relationship between the pixels (i.e. the interferograms on the detector) and the observables (i.e. the complex visibilities). In other words, the AMBER data reduction algorithm is based on modelling the interferogram in the detector plane.

In Sect. 2, we present the AMBER experiment from a signalprocessing point of view and we introduce the interferometric equation governing this instrument. We develop the specific data reduction processes of AMBER in Sect. 3, and then derive the estimators of the interferometric observables. Successive steps in the data reduction method are given in Sect. 4, as performed by the software provided to the community. Finally, the datareduction algorithm is validated in Sect. 5 through several "onsky" observations with the VLTI (commissioning and science demonstration time (SDT)). Present and future performances of this instrument are discussed.

\section{Presentation of the instrument}

\subsection{Image formation}

The process of image formation of AMBER is sketched in Fig. 1 (left) from a signal-processing point of view. It consists of three major steps. First, the beams from the three telescopes are filtered by single-mode fibers to convert phase fluctuations of the corrugated wavefronts into intensity fluctuations that are monitored. The fraction of light entering the fiber is called the coupling coefficient (Shaklan \& Roddier 1988) and it depends on the Strehl ratio (Coudé du Foresto et al. 2000). At this point, a pair of conjugated cylindrical mirrors compresses, by a factor 
Table 1. Detector properties.

\begin{tabular}{cc}
\hline \hline \multicolumn{2}{c}{ Detector specifications } \\
\hline Society/Name & Rockwell/HAWAII \\
Composition & HgCdTe \\
Number of pixels & $512 \times 512$ \\
Pixel size & $18.5 \mu \mathrm{m} \times 18.5 \mu \mathrm{m}$ \\
Spectral width & $0.8 \mu \mathrm{m}-2.5 \mu \mathrm{m}$ \\
Readout noise & $9 \mathrm{e}^{-}$ \\
$\mathrm{e}^{-} /$ADU & 4.18 \\
Cooling & Liquid nitrogen \\
Temperature & $78 \mathrm{~K}$ \\
Autonomy of cryostat & $24 \mathrm{~h}$ \\
\hline
\end{tabular}

of about 12 , the individual beams exiting from fibers into one dimensional elongated beams to be injected in the entrance slit of the spectrograph. For each of the three beams, beam-splitters placed inside the spectrograph select part of the light and induce three different tilt angles so that each beam is imaged at different locations of the detector. These are called photometric channels and are each one relative to a corresponding incoming beam. The remaining parts of the light of the three beams are overlapped on the detector image plane to form fringes. The spatial coding frequencies of the fringes $f$ are fixed by the separation of the individual output pupils. They are $f=[1,2,3] d / \lambda$, where $d$ is the output pupil diameter. Since the beams hit a spectral dispersing element (a prism glued on a mirror or one of the two gratings) in the pupil plane, the interferogram and the photometries are spectrally dispersed perpendicularly to the spatial coding. The dispersed interferogram arising from the beam combination, as well as the photometric outputs are recorded on the infrared detector, which characteristics are given in Table 1 .

The detector consists in a $512 \times 512$ pixel array with the vertical dimension aligned with the wavelength direction. The first 20 pixels of each scanline of the detector are masked and never receive any light, allowing us to estimate the readout noise and bias during an exposure. The light from the two (resp. 3) telescopes comes in three (resp. 4) beams, one "interferometric" beam where the interference fringes are located, and two (resp. three) "photometric" beams. These 3 (resp. 4) beams are dispersed and spread over three (resp. 4) vertical areas on the detector. The detector is read in subwindows. Horizontally, these subwindows are centered on the regions where the beams are dispersed, with a typical width of 32 to 40 pixels. Vertically, the detector can be set up to read up to three subwindows (covering up to three different wavelength ranges). The raw data format used by AMBER records individually these subframes. However, as sketched in the right panel of Fig. 1, the AMBER raw data can be conceived as the grouping together of these subwindows:

- The left column (noted "DK") contains the masked pixels.

- The two following columns (noted "P1" and "P2") and the right one in the three telescope mode (noted "P3"), usually of 32 pixels wide, are the photometric outputs. They record the photometric signal coming from the three telescopes. When dealing with 2-telescope observations, only channels P1 and P2 are lit.

- The fourth column is the interferometric output (denoted "IF"). it exhibits the interference fringes arising from the recombination of the beams (that is, two or three beams, according to the number of telescopes used). We call $N_{\text {pix }}$ the number of pixels in this column, which is usually $N_{\text {pix }}=32$.
The individual image that is recorded during the detector integration time (DIT) is called a frame. A cube of frames obtained during the exposure time is called an exposure.

\subsection{AMBER interferometric equation}

The following demonstration is given considering a generic $N_{\text {tel }} \geq 2$ telescope interferometer. In the specific case of AMBER, however, $N_{\text {tel }}=2$ or $N_{\text {tel }}=3$. Each line of the detector being independent of each other, we can focus our attention on one single spectral channel ${ }^{1}$, which is assumed to be monochromatic here. The effect of a spectral bandwidth on the interferometric equation is treated in Sect. 3.6.1.

Interferometric output: when only the $i$ th beam is illuminated, the signal recorded in the interferometric channel is the photometric flux $F^{i}$ spread on the Airy pattern $a_{k}^{i}$, which is the diffraction pattern of the $i$ th output pupil weighted by the single-mode of the fiber, $k$ is the pixel number on the detector, and $\alpha$ is the associated angular variable. Then, $F^{i}$ results in the total source photon flux $N$ attenuated by the total transmission of the $i$ th optical train $t^{i}$, i.e. the product of the optical throughput (including atmosphere and optical train of the VLTI and the instrument) and the coupling coefficient of the single-mode fiber:

$F^{i}=N t^{i}$.

When beams $i$ and $j$ are illuminated simultaneously, the coherent addition of both beams results in an interferometric component superimposed on the photometric continuum. The interferometric part, i.e. the fringes, arises from the amplitude modulation of the coherent flux $F_{\mathrm{c}}^{i j}$ at the coding frequency $f^{i j}$. The coherent flux is the geometrical product of the photometric fluxes, weighted by the visibility:

$F_{\mathrm{c}}^{i j}=2 N \sqrt{t^{i} t^{j}} V^{i j} \mathrm{e}^{i\left(\Phi^{i j}+\phi_{\mathrm{p}}^{i j}\right)}$

where $V^{i j} \mathrm{e}^{i \Phi^{i j}}$ is the complex modal visibility (Mège et al. 2003) and $\phi_{\mathrm{p}}^{i j}$ takes a potential differential atmospheric piston into account. Note that, strictly speaking, the modal visibility is not the source visibility. However, the study of the relation between the modal visibility and the source visibility is beyond the scope of this paper, but further information can be found in Mège et al. (2003) and Tatulli et al. (2004). Here we consider our observable to be the complex modal visibility.

Such an analysis can be done for each pair of beams arising from the interferometer. As a result, the interferogram recorded on the detector can be written in the general form:

$i_{k}=\sum_{i}^{N_{\text {tel }}} a_{k}^{i} F^{i}+\sum_{i<j}^{N_{\text {tel }}} \sqrt{a_{k}^{i} a_{k}^{j}} C_{\mathrm{B}}^{i j} \operatorname{Re}\left[F_{\mathrm{c}}^{i j} \mathrm{e}^{i\left(2 \pi \alpha_{k} f^{i j}+\phi_{\mathrm{s}}^{i j}+\Phi_{\mathrm{B}}^{i j}\right)}\right]$.

Here, $\phi_{\mathrm{s}}^{i j}$ is the instrumental phase taking possible misalignment and/or differential phase between the beams $a_{k}^{i}$ and $a_{k}^{j}$ into account, and $C_{\mathrm{B}}^{i j}$ and $\Phi_{\mathrm{B}}^{i j}$ are, respectively, the loss of contrast and the phase shift due to polarization mismatch between the two beams (after the polarizers), such as the rotation of the single-mode fibers might induce. This equation is governing the AMBER fringe pattern, that is the interferometric channel

1 In practice, there is a previous image-centering step, where each channel is re-centered with respect to the others along the wavelength dimension, as explained in Sect. 4. 
of the fourth column. The first sum in Eq. (3), which represents the continuum part of the interference pattern, is called the DC component from now on, and the second sum, which describes the high frequency part (that is the coded fringes), is called the AC component of the interferometric output.

Photometric outputs: thanks to the photometric channels, the number of photoevents $p^{i}(\alpha)$ coming from each telescope can be estimated independently with

$p_{k}^{i}=F^{i} b_{k}^{i}$

where $b_{k}^{i}$ is the beam profile in the $i$ th photometric channel. The previous equation rules the photometric channels.

\section{Data reduction algorithm}

The AMBER data-reduction algorithm is based on the modelling of the interferogram in the detector plane. Such a method requires an accurate calibration of the instrument.

\subsection{Modelling the interferogram}

In order to model the interferogram, we distinguish between the astrophysical and instrumental parts in the interferometric equation. It becomes:

$i_{k}=\sum_{i}^{N_{\mathrm{tel}}} a_{k}^{i} F^{i}+\sum_{i<j}^{N_{\mathrm{tel}}}\left[c_{k}^{i j} R^{i j}+d_{k}^{i j} I^{i j}\right]$

with

$$
\begin{gathered}
c_{k}^{i j}=C_{\mathrm{B}}^{i j} \frac{\sqrt{a_{k}^{i} a_{k}^{j}}}{\sqrt{\sum_{k} a_{k}^{i} a_{k}^{j}}} \cos \left(2 \pi \alpha_{k} f^{i j}+\phi_{\mathrm{s}}^{i j}+\Phi_{\mathrm{B}}^{i j}\right), \\
d_{k}^{i j}=C_{\mathrm{B}}^{i j} \frac{\sqrt{a_{k}^{i} a_{k}^{j}}}{\sqrt{\sum_{k} a_{k}^{i} a_{k}^{j}}} \sin \left(2 \pi \alpha_{k} f^{i j}+\phi_{\mathrm{s}}^{i j}+\Phi_{\mathrm{B}}^{i j}\right),
\end{gathered}
$$

and

$R^{i j}=\sqrt{\sum_{k} a_{k}^{i} a_{k}^{j}} \operatorname{Re}\left[F_{\mathrm{c}}^{i j}\right], I^{i j}=\sqrt{\sum_{k} a_{k}^{i} a_{k}^{j}} \operatorname{Im}\left[F_{\mathrm{c}}^{i j}\right]$.

As an analogy with telecom data processing, $c_{k}^{i j}$ and $d_{k}^{i j}$ are called the carrying waves of the signal at the coding frequency $f^{i j}$, since they carry (in terms of amplitude modulation) $R^{i j}$ and $I^{i j}$, which are directly linked to the complex coherent flux (as shown by Eq. (8)).

The estimated photometric fluxes $P^{i}$ are computed from the photometric channels (see Eq. (4)):

$P^{i}=F^{i} \sum_{k} b_{k}^{i}$

If we know the ratio $v_{k}^{i}$ - which only depends of the instrumental configuration - between the measured photometric fluxes $P^{i}$ and the corresponding DC components of the interferogram, we can have an estimation of the latter thanks to the following formula:

$a_{k}^{i} F^{i}=P^{i} v_{k}^{i}$
Table 2. Acquisition sequence of calibration files.

\begin{tabular}{cccccc}
\hline \hline Step & Sh 1 & Sh 2 & Sh 3 & Phase $\gamma_{0}$ & DPR key \\
\hline 1 & O & X & X & NO & 2P2V, 3P2V \\
2 & X & O & X & NO & 2P2V, 3P2V \\
3 & O & O & X & NO & 2P2V, 3P2V \\
4 & O & O & X & YES & 2P2V, 3P2V \\
\hline 5 & X & X & O & NO & 3P2V \\
6 & O & X & O & NO & 3P2V \\
7 & O & X & O & YES & 3P2V \\
8 & X & O & O & NO & 3P2V \\
9 & X & O & O & YES & 3P2V \\
\hline
\end{tabular}

$\mathrm{Sh}=$ Shutter $\mathrm{O}=$ Open; $\mathrm{X}=$ Closed .

We then can compute the DC continuum corrected interferogram $m_{k}$,

$m_{k}=i_{k}-\sum_{i=1}^{N_{\text {tel }}} P^{i} v_{k}^{i}$

which can be rewritten:

$m_{k}=c_{k}^{i j} R^{i j}-d_{k}^{i j} I^{i j}$

This equation defines a system of $N_{\text {pix }}$ linear equations with $2 N_{\mathrm{b}}=N_{\text {tel }}\left(N_{\text {tel }}-1\right)$ unknowns (i.e. twice the number of baselines). It characterizes the linear link between the pixels on the detector and the complex visibility:

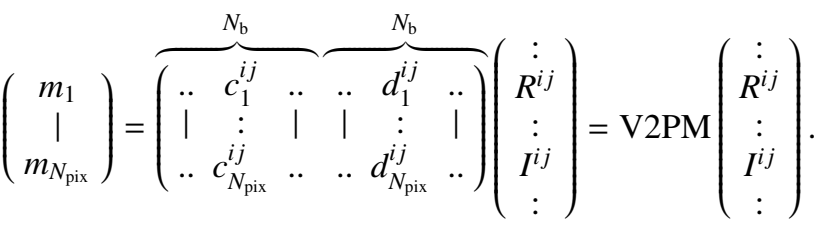

The V2PM matrix (namely visibility to pixel matrix), which contains the carrying waves, holds the information about the interferometric beams $\sqrt{a_{k}^{i} a_{k}^{j}}$, the coding frequencies $f^{i j}$, and the instrumental differential phases $\phi_{\mathrm{s}}^{i j}$. Together with the $v_{k}^{i}$, they entirely describe the instrument from a signal processing point of view. These quantities, namely $c_{k}^{i j}, d_{k}^{i j}$, and $v_{k}^{i}$, have, however, to be calibrated.

\subsection{Calibration procedure}

The calibration procedure is performed thanks to an internal source located in the Calibration and Alignment Unit (CAU) of AMBER (Petrov et al. 2007). It consists of acquiring a sequence of high signal-to-noise ratio calibration files, whose successive configurations are summarized in Table 2 and explained below. Since the calibration is done in laboratory, the desired level of accuracy for the measurements is insured by choosing the appropriate integration time. As an example, typical integration times in "average accuracy" mode are (for the full calibration process) $\tau=17 \mathrm{~s}, 30 \mathrm{~s}, 800 \mathrm{~s}$ for, respectively, low, medium, and high spectral resolution modes in the $\mathrm{K}$ band and 100 times higher for the "high accuracy" calibration mode.

The sequence of calibration files has been chosen to accommodate both two and three-telescope operations. For a twotelescope operation, only the 4 first steps are needed. Raw data FITS files produced by the ESO instruments bear no identifiable 

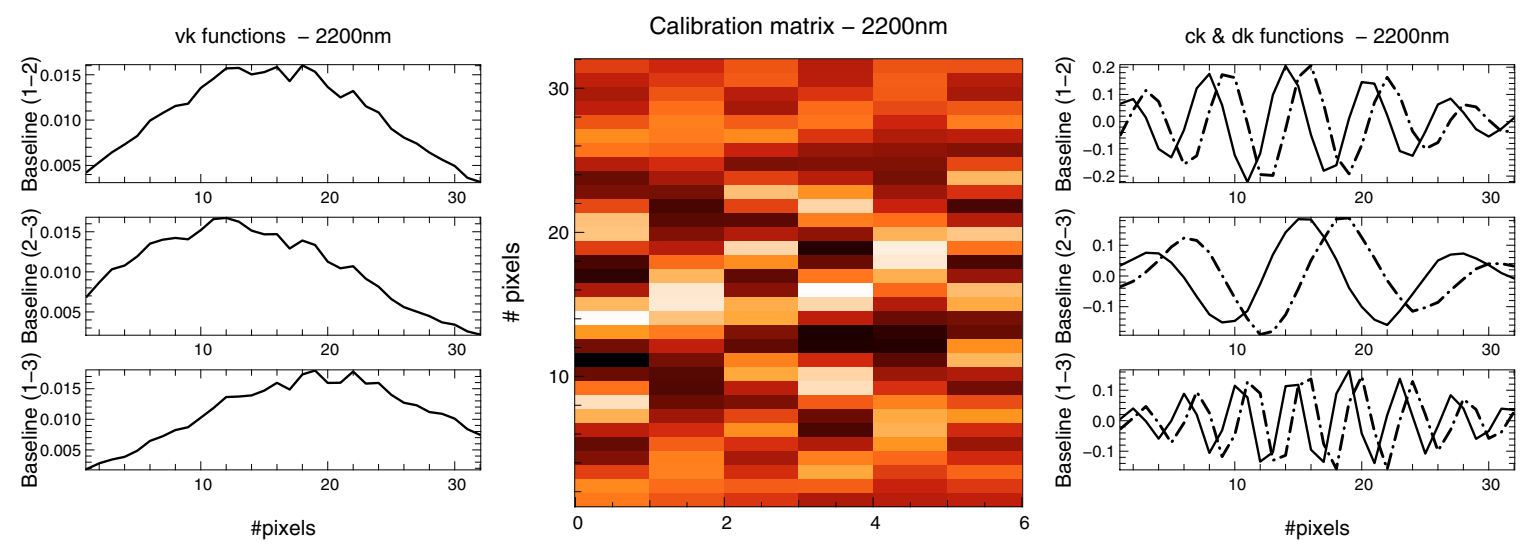

Fig. 2. Outputs of the calibration procedures. Examples have been chosen for one given wavelength: $\lambda=2.2 \mu \mathrm{m}$. Left: the $v_{k}^{i}$ functions. Middle: the matrix containing the carrying waves; the first three columns are the $c_{k}^{i j}$ functions for each baseline, and the three last columns are the respective $d_{k}^{i j}$ functions. One can see that for each baseline $c_{k}^{i j}$ and $d_{k}^{i j}$ are in quadrature. Right: another representation of the carrying waves. From top to bottom, both sinusoidal functions correspond to columns $1-4,2-5$, and $3-6$ of the calibration matrix.

name and can only be identified as, e.g., files relevant to the calibration of the V2PM matrix, by the presence of dedicated FITS keywords (ESO's pipeline Data PRoduct keys or "DPR keys") in their header. The DPR keys used are listed in Table 2.

First (steps 1 and 2 - and 5 when in 3-telescope mode), for each telescope beam, an image is recorded with only this shutter opened. The fraction of flux measured between the interferometric channel and the illuminated photometric channel leads to an accurate estimation of the $v_{k}^{i}$ functions. Then, in order to compute the carrying waves $c_{k}^{i j}$ and $d_{k}^{i j}$, one needs to have two independent (in terms of algebra) measurements of the interferogram since there are two unknowns (per baseline) to compute. The principle is the following: two shutters are opened simultaneously (steps 3/4,6/7, and 8/9) and for each pair of beams, then the interferogram is recorded on the detector. Such an interferogram corrected for its DC component and calibrated by the photometry yields the knowledge of the $c_{k}^{i j}$ carrying wave. To obtain its quadratic counterpart, the previous procedure is repeated by introducing a known phase shift close to 90 degree $\gamma_{0}$ using piezoelectric mirrors at the entrance of beams 2 and 3. Computing the $d_{k}^{i j}$ function from the knowledge of $c_{k}^{i j}$ and $\gamma_{0}$ is straightforward. Note that by construction: (i) the carrying waves are computed with the unknown system phase $\Phi^{c}$ (possible phase of the internal source, differential optical path difference introduced at the CAU level, etc.), and (ii) since the internal source in the CAU is slightly resolved by the largest baseline (1-3) of the output pupils, the carrying waves for this specific baseline are weighted by the visibility $V_{\mathrm{c}}$ of the internal source. Hence, at this point, the carrying waves are following expressions that are slightly different from their original definition given by Eqs. (6) and (7):

$$
\begin{gathered}
c_{k}^{i j}=\frac{\sqrt{a_{k}^{i} a_{k}^{j}}}{\sqrt{\sum_{k} a_{k}^{i} a_{k}^{j}}} C_{\mathrm{B}}^{i j} V_{\mathrm{c}}^{i j} \cos \left(2 \pi \alpha_{k} f^{i j}+\phi_{\mathrm{s}}^{i j}+\Phi_{\mathrm{B}}^{i j}+\Phi_{\mathrm{c}}^{i j}\right) \\
d_{k}^{i j}=\frac{\sqrt{a_{k}^{i} a_{k}^{j}}}{\sqrt{\sum_{k} a_{k}^{i} a_{k}^{j}}} C_{\mathrm{B}}^{i j} V_{\mathrm{c}}^{i j} \sin \left(2 \pi \alpha_{k} f^{i j}+\phi_{\mathrm{s}}^{i j}+\Phi_{\mathrm{B}}^{i j}+\Phi_{\mathrm{c}}^{i j}\right) .
\end{gathered}
$$

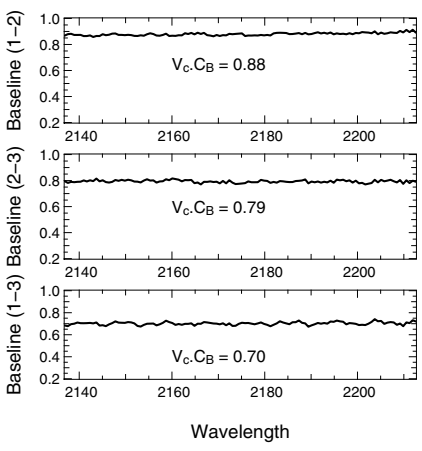

Fig. 3. Contrast loss due to polarization effects and partial resolution of the internal source as a function of the wavelength. The 3-telescope P2VM used is the same as the one presented in Fig. 2. The errors bars are roughly at the level of the contrast loss rms along the wavelength. In other words, the contrast loss is constant over the wavelength range.

However, since $c_{k}^{i j}$ and $d_{k}^{i j}$ are shifted by $\pi / 2$, they insure the following relation:

$$
\sum_{k}^{N_{\mathrm{pix}}} c_{k}^{2}+d_{k}^{2}=C_{\mathrm{B}}^{2} V_{\mathrm{c}}^{2} .
$$

Hence, the conjugated loss of visibility due to the internal source and the polarization effects can be known and calibrated ${ }^{1}$ by computing the previous formula. Unfortunately, since it is not possible to disentangle both contrast losses, and since the $V_{\mathrm{c}}$ factor only affects the interferograms arising from the calibration procedure, and not from the observation, the visibility estimated on a star will be affected from this factor as well, as shown in Sect. 3.5.1.

Figure 3 illustrates Eq. (16). For the baselines $(1,2)$ and $(2,3)$, the contrast loss arises from polarization effects, since the internal source is unresolved. We find $C_{\mathrm{B}}^{12} \simeq 0.9$ and $C_{\mathrm{B}}^{23} \simeq 0.8$, respectively. For the third baseline $(1,3)$, the internal source is partially resolved, which explains an higher contrast loss, $C_{\mathrm{B}}^{13} V_{\mathrm{c}}^{13} \simeq 0.7$.

1 This step is not yet provided in the amdlib software described in Sect. 4. 


\subsection{Fringe fitting}

To estimate the coherent fluxes $R^{i j}$ and $I^{i j}$, which are at the basis of the computation of the whole AMBER observables, one has to solve the inverse problem described by Eq. (13), i.e. one has to perform an $\chi^{2}$ linear fit of the fringes, with the coherent fluxes being the unknown parameters. The solution is given by the following equation

$\left[\widetilde{R}^{i j}, \widetilde{I}^{i j}\right]=\mathrm{P} 2 \mathrm{VM}\left[m_{k}\right]$,

where

$\mathrm{P} 2 \mathrm{VM}=\left[\mathrm{V} \mathrm{PM}^{\mathrm{T}} C_{\mathrm{M}}^{-1} \mathrm{~V} 2 \mathrm{PM}\right]^{-1} \mathrm{~V} \mathrm{PM}^{\mathrm{T}} C_{\mathrm{M}}^{-1}$

is the generalized inverse of the V2PM matrix, $C_{\mathrm{M}}$ being the covariance matrix of the measurements $m_{k}$, and $X^{\mathrm{T}}$ denoting the transpose of the $X$ matrix. P2VM means Pixel to Visibility Matrix since it allows estimation of the complex visibility from the interferogram recorded on the detector. Assuming that the pixels on the detector are uncorrelated, the $C_{M}$ matrix is diagonal, with each term of the diagonal defined by the variance of the DC-corrected interferogram $\sigma^{2}\left(m_{k}\right)$. The fundamental error on the DC corrected interferogram arises from the photon noise and detector noise (of variance $\sigma$ ) corrupting the measurements, that is, each pixel of the interferogram $i_{k}$ and the estimated photometric fluxes $P^{i}$. It becomes:

$\sigma^{2}\left(m_{k}\right)=\overline{i_{k}}+\sigma^{2}+\sum_{i=1}^{N_{\text {tel }}}\left[\overline{P_{i}}+N_{\text {pix }} \sigma^{2}\right]\left(v_{k}^{i}\right)^{2}$.

\subsection{Fringe detection}

Positive detection of fringes in the measurements requires, at the same time, enough flux entering the fibers and high enough fringe contrast, so that the fringes rise with the noise level. As a result, the computation of the signal-to-noise ratio of the coherent flux, which takes both of the parameters into account, appears naturally as the relevant criterion to use.

$S N R^{2}(t)=\frac{1}{N_{\mathrm{b}}} \frac{1}{N_{l}} \sum_{b}^{N_{\mathrm{b}}} \sum_{l}^{N_{l}}\left[\left(\frac{R^{b^{2}}(l, t)}{\sigma_{R^{b}}^{2}}-1\right)+\left(\frac{I^{b^{2}}(l, t)}{\sigma_{I^{b}}^{2}}-1\right)\right]$

$b$ being for sake of simplicity the baseline number that describes each pair of telescopes $(i, j), N_{\mathrm{b}}$ the number of baselines, and $N_{l}$ the number of spectral channels. In the absence of fringes, the quantities $R^{b^{2}}$ and $I^{b^{2}}$ tend toward $\sigma_{R^{b}}^{2}$ and $\sigma_{I^{b}}^{2}$, respectively, thus driving the fringe criterion toward 0 . In contrast, the presence of fringes above the noise level, that is, when $R^{b^{2}}(l, t)>\sigma_{R^{b}}^{2}$ and/or $I^{b^{2}}(l, t)>\sigma_{I^{b}}^{2}$ imposes the fringe criterion to be strictly superior to 0 and is directly linked to the quality of the frames. It thus allows us to operate a fringe selection prior to the proper estimation of the observables, a step that can be useful for sets of data recorded in bad observational conditions, as shown in Sect. 5.2.

The values $\sigma_{R^{b}}^{2}$ and $\sigma_{I^{b}}^{2}$, the bias part of $R^{2}$ and $I^{2}$, can be easily computed from the definition of the real and imaginary part of the coherent fluxes, which are linear combinations of the DC continuum-corrected interferograms $m_{k}$. If $\zeta_{k}^{b}$ and $\xi_{k}^{b}$ are the coefficients of the P2VM matrix, $R^{b}$ and $I^{b}$ verify the respective following equations:

$R^{b}=\sum_{k=1}^{N_{\mathrm{pix}}} \zeta_{k}^{b} m_{k}, \quad I^{b}=\sum_{k=1}^{N_{\mathrm{pix}}} \xi_{k}^{b} m_{k}$.
It is then straightforward that

$$
\sigma_{R^{b}}^{2}=\sum_{k}\left(\zeta_{k}^{b}\right)^{2} \sigma^{2}\left(m_{k}\right) ; \sigma_{I^{b}}^{2}=\sum_{k}\left(\xi_{k}^{b}\right)^{2} \sigma^{2}\left(m_{k}\right)
$$

\subsection{Estimation of the observables}

For each spectral channel, the squared visibility and closure phase (in the three telescope case) can be estimated from the interferogram. Taking advantage of the spectral dispersion, the differential phase can be computed as well. In the following paragraphs, we denote with $\langle\ldots\rangle$ the ensemble average of the different quantities. This average can be performed either on the frames within an exposure and/or on the wavelengths.

\subsubsection{The squared visibility}

Theoretically speaking, the squared visibility is given by computing the ratio between the squared coherent flux and the photometric fluxes. Following Eqs. (1), (2), (8) and (9) it becomes:

$\frac{\left|F_{\mathrm{c}}^{i j}\right|^{2}}{4 F^{i} F^{j}}=\frac{R^{i j^{2}}+I^{i j^{2}}}{4 P^{i} P^{j} \sum_{k} v_{k}^{i} v_{k}^{j}}=\frac{\left|V^{i j}\right|^{2}}{V_{\mathrm{c}}^{i j^{2}}}$.

Note that, thanks to the calibration process, the computed visibility is free of the instrumental contrast, which is the loss of contrast due to the instrument; but as mentioned in Sect. 3.2, the object visibility is weighted by the visibility of the internal source. However, this factor $\left(V_{\mathrm{c}}^{i j^{2}}\right)$ automatically disappears when doing the necessary atmospheric calibration (see Sect. 3.6.2).

As a result the visibility - atmospheric issues apart - has still to be calibrated by observing a reference source. In practice, because data are noisy, we perform an ensemble average on the frames that compose the data cube (see Sect. 2.1) to estimate the expected values of the square coherent flux and the photometric fluxes, respectively. Taking the average of the squared modulus of the coherent flux, i.e. doing a quadratic estimation, allows us to handle the problem of the random differential piston $\phi_{\mathrm{p}}^{i j}$, but introduces a quadratic bias due to the zero-mean photon and detector noises (Perrin 2003). The expression of the squared visibility estimator, unbiased by fundamental noises is therefore:

$\frac{\left|\widetilde{V^{i j}}\right|^{2}}{V_{\mathrm{c}}^{i j^{2}}}=\frac{\left\langle R^{i j^{2}}+I^{i j^{2}}\right\rangle-\operatorname{Bias}\left\{R^{i j^{2}}+I^{i j^{2}}\right\}}{4\left\langle P^{i} P^{j}\right\rangle \sum_{k} v_{k}^{i} v_{k}^{j}}$.

The quadratic bias of the squared amplitude of the coherent flux writes as the quadratic sum of the biases of $R^{2}$ and $I^{2}$. From Eq. (22), we get:

$\operatorname{Bias}\left\{R^{i j^{2}}+I^{i j^{2}}\right\}=\sum_{k}\left[\left(\zeta_{k}^{i j}\right)^{2}+\left(\xi_{k}^{i j}\right)^{2}\right] \sigma^{2}\left(m_{k}\right)$

The previous equation is nothing but the mathematical expression that describes the bias as the quadratic sum of the errors of the measurements $\sigma^{2}\left(m_{k}\right)$ (as defined by Eq. (19)) projected on the real and imaginary axis of the coherent flux.

Using the squared visibility estimator of Eq. (24), the theoretical error bars on the squared visibility can be computed from its second-order Taylor expansion (Papoulis 1984; Kervella et al. 2004):

$\sigma^{2}\left(\left|V^{i j}\right|^{2}\right)=\frac{1}{M}\left[\frac{\sigma^{2}\left(\left|C^{i j}\right|^{2}\right)}{{\overline{\left.C_{i j}\right|^{2}}}^{2}}+\frac{\sigma^{2}\left(P^{i} P^{j}\right)}{{\overline{P^{i} P^{j}}}^{2}}\right]{\overline{\left|V^{i j}\right|^{2}}}^{2}$ 
where $\left|C^{i j}\right|^{2}=R^{i j^{2}}+I^{i j^{2}}-\operatorname{Bias}\left\{R^{i j^{2}}+I^{i j^{2}}\right\}$ is the unbiased squared coherent flux. In practice, the expected value and the variance of the squared coherent flux and the photometric fluxes are computed empirically from the $M$ available measurements. We then get the following semi-empirical formula:

$$
\begin{aligned}
\sigma_{\text {stat }}^{2}\left(\mid \widetilde{\left.V^{i j}\right|^{2}}\right)= & \frac{1}{M}\left[\frac{\left\langle\left|C^{i j}\right|^{4}\right\rangle_{\mathrm{M}}-\left\langle\left|C^{i j}\right|^{2}\right\rangle_{\mathrm{M}}^{2}}{\left\langle\left|C^{i j}\right|^{2}\right\rangle_{\mathrm{M}}^{2}}\right. \\
& \left.+\frac{\left\langle P^{i^{2}} P^{j^{2}}\right\rangle_{\mathrm{M}}-\left\langle P^{i} P^{j}\right\rangle_{\mathrm{M}}^{2}}{\left\langle P^{i} P^{j}\right\rangle_{\mathrm{M}}^{2}}\right] \mid{\widetilde{V^{i j}}}^{2}
\end{aligned}
$$

Note finally that, although quadratic estimation of the visibility has been computed, the squared visibility will be systematically decreased by the atmosphere jitter during the frame integration time. We focus on this effect in Sect. 3.6.

\subsubsection{The closure phase}

By definition, the closure phase is the phase of the so-called bispectrum $B^{123}$. The bispectrum results in the ensemble average of the coherent flux triple product and then estimated as

$\widetilde{B}^{123}=\left\langle C^{12} C^{23} C^{13^{*}}\right\rangle$

where $C^{i j}=R^{i j}+i I^{i j}$. The closure phase then is straightforward:

${\widetilde{\phi_{B}}}^{123}=\operatorname{atan}\left[\frac{\operatorname{Im}\left(\widetilde{B}^{123}\right)}{\operatorname{Re}\left(\widetilde{B}^{123}\right)}\right]$.

The closure phase presents the advantage of being independent of the atmosphere (e.g. Roddier 1986). However in the case of AMBER, the closure phase of the image might not coincide with the one of the object and might be biased because of the calibration process. If the so-called system phase presents a non-zero closure phase $\Phi_{\mathrm{c}}^{12}+\Phi_{\mathrm{c}}^{23}-\Phi_{\mathrm{c}}^{13}$, this bias must be calibrated by observing a point source or at least a centro-symmetrical object. So far, no theoretical computation of the error of the closure phase has been provided for the AMBER data-reduction algorithm. Thus, closure-phase internal error bars (i.e. that does not include systematics errors) are computed statistically by taking the root mean square of all the individual frames, then dividing by the square root of the number of frames, as illustrated in Sect. 5.5.

\subsubsection{The differential phase}

The differential phase is the phase of the so-called cross spectrum $W_{12}$. For each baseline, the latter is estimated from the complex coherent flux taken at two different wavelengths $\lambda_{1}$ and $\lambda_{2}$ :

$\widetilde{W_{12}^{i j}}=\left\langle C_{\lambda_{1}}^{i j} C_{\lambda_{2}}^{i j^{*}}\right\rangle$

And the differential phase is:

$\widetilde{\Delta \phi_{12}^{i j}}=\operatorname{atan}\left[\frac{\operatorname{Im}\left(\widetilde{W_{12}^{i j}}\right)}{\operatorname{Re}\left(\widetilde{W_{12}^{i j}}\right)}\right]$.

\subsubsection{The piston}

The interferometric phase induced by the achromatic piston term takes the form

$\phi_{\lambda}^{i j}=\frac{2 \pi \delta^{i j}}{\lambda}=2 \pi \delta^{i j} \sigma$

where $\delta^{i j}$ is the achromatic differential piston between telescope $i$ and $j, \lambda$ is the wavelength, and $\sigma$ is the wavenumber (i.e. $\sigma=1 / \lambda$ ).

First order Taylor expansion: at first order, the estimated differential phase of Eq. (31) is a linear function that takes the generic form $\Delta \phi_{12}=\phi_{1}+2 \pi\left(\sigma_{2}-\sigma_{1}\right) \delta$. Its slope $\delta$ depends on the sum of atmospheric piston $\delta_{\mathrm{p}}$, which varies frame by frame, and of the linear component of the object differential phase $\delta_{\mathrm{o}}$. A good estimate of this slope in the presence of noise is the argument of the average cross spectrum along the wavelengths:

$\widetilde{\delta_{\mathrm{p}}^{i j}}+\widetilde{\delta_{\mathrm{o}}^{i j}}=\frac{\arg \left\langle W_{\lambda_{2 l}, \lambda_{2 l+1}}^{i j}\right\rangle_{l}}{2 \pi\left\langle\sigma_{\lambda_{2 l+1}}-\sigma_{\lambda_{2 l}}\right\rangle_{l}}$.

Estimation of the piston is unbiased when the wave number varies linearly with the spectral pixel index (linear grating dispersion law). This can be true with an excellent approximation at medium spectral resolution and high spectral resolution in the AMBER case. However, for the low spectral resolution, biases as high as $5 \%$ in the estimation of piston can occur.

Fitting the complex phasor: the achromatic piston can also be estimated from a least-square fit of the complex coherent flux. If we define the complex phasor as

$\Psi_{\lambda}=C_{\lambda}^{i j} \times \mathrm{e}^{\frac{2 i \pi \delta^{i j}}{\lambda}}$,

$\delta^{i j}$ can be retrieved by minimizing the phase of such complex phasoror, equivalently, by minimizing the tangent of the phase. The $\chi^{2}$ is then defined as:

$\chi^{2}=\frac{\sum_{\lambda} \frac{\left(\frac{\operatorname{Im}\left(\Psi_{\lambda}\right)}{\operatorname{Re}\left(\Psi_{\lambda}\right)}\right)^{2}}{\sigma_{R_{\lambda}}^{2}+\sigma_{I_{\lambda}}^{2}}}{\sum_{\lambda} \frac{1}{\sigma_{R_{\lambda}}^{2}+\sigma_{I_{\lambda}}^{2}}}$.

This $\chi^{2}$ is highly non-linear and simple techniques such as gradient fitting cannot be used here. On the contrary, non-linear fitting techniques such as genetic or simulated annealing algorithms (Kirkpatrick et al. 1983) must be used instead.

Note that, in order to distinguish between the atmospheric piston $\delta_{\mathrm{p}}$ and the linear component of the differential phase $\delta_{\mathrm{o}}$, the fitting techniques described above can be performed by only using spectral channels corresponding to the continuum of the source (i.e. outside spectral features) where its differential phase of the object is assumed to be zero.

\subsection{Biases of the visibility}

\subsubsection{Loss of spectral coherence}

The above derivation of the interferometric equation assumes a monochromatic spectral channel. In practice, the spectral width of one spectral channel is non zero and depends on the resolution $\mathcal{R}$ of the spectrograph. As a consequence the coherence 
length $\mathcal{L}_{\mathrm{c}}$ of the interferogram is finite and equals $\mathcal{L}_{\mathrm{c}}=\lambda_{0} \mathcal{R}$, where $\lambda_{0}$ is the reference wavelength in the spectral channel. Assuming a linear decomposition of the phase of the interferogram and neglecting higher orders, the interferogram is attenuated by a factor $\rho_{k}$, which can be written

$\rho_{k}=\left|\widehat{\mathcal{F}}\left(\pi \frac{\delta_{k}+\delta_{\mathrm{p}}+\delta_{\mathrm{o}}}{\mathcal{L}_{\mathrm{c}}}\right)\right|$,

where $\widehat{\mathcal{F}}$ is the Fourier transform of the spectral filter function, $\delta_{k}$ is the spatial sampling of the interferogram (that is, the pixel coordinates expressed in optical path difference (OPD) units), $\delta_{\mathrm{p}}$ and $\delta_{\mathrm{o}}$ the atmospheric piston and the slope of the object spectral differential phase, respectively, as defined in Sect. 3.5.4. Note that for a square filter, the attenuation coefficient takes the well-known form of the sinc function:

$\rho_{k}=\left|\operatorname{sinc}\left(\pi \frac{\delta_{k}+\delta_{\mathrm{p}}+\delta_{\mathrm{o}}}{\mathcal{L}_{\mathrm{c}}}\right)\right|$.

In the low-resolution mode where $\mathcal{R}=35$, the attenuation coefficient strongly depends on the pixel position $\delta_{k}$, which is calibratable quantity. Nonetheless, the compensation for this effect requires an iterative process in two steps where (i) the estimation of $\delta_{\mathrm{p}}+\delta_{\mathrm{o}}$ is performed as described in Sect. 3.5.4 and (ii) the $\rho_{k}$ attenuation correction is applied directly to the DC corrected interferograms $m_{k}$. The loop is then repeated until convergence. This algorithm, which has not yet been implemented in the software, will be described in greater detail in a forthcoming paper.

In the medium and high resolutions (where $\mathcal{R}=1500$ and $\mathcal{R}=10000$, respectively), however, the OPD $\delta_{k}$ due the spatial sampling of AMBER can be neglected. Indeed this approximation leads to a relative error of the coefficient below $10^{-3}$ and $10^{-5}$, respectively, which is within the specified error bars of the visibility. In such a case, the loss of spectral coherence simply results in biasing the visibility frame to frame by a factor $\rho\left(\delta_{\mathrm{p}}+\delta_{\mathrm{o}}\right)$. This bias can be corrected by knowing the shape of the spectral filter and by estimating the piston $\delta_{\mathrm{p}}+\delta_{\mathrm{o}}$ thanks to Eq. (33).

\subsubsection{Atmospheric jitter}

Although a quadratic estimation of the visibility has been performed to avoid the differential piston to completely cancel out the fringes, the high frequency variations of the latter during the integration time - so called high-pass jitter - nevertheless blur the fringes. As a result, the coherent flux, thus the visibility, is attenuated. On average, the attenuation coefficient $\Gamma$ of the squared visibility is given by Colavita (1999b):

$\Gamma=\exp \left(-\sigma_{\phi_{h f}^{p}}^{2}\right)$

where $\sigma_{\phi_{h f}^{p}}^{2}$ is the variance of the high-pass jitter $\phi_{h f}^{p}$.

For the time being, this atmospheric effect is compensated by calibrating the source visibility with a reference source observed shortly before and after the scientific target to insure similar atmospheric conditions. We have also planned in the near future to provide a more accurate calibration of this effect, based on computing the variance of the so-called "first difference phase jitter", which is the difference of the average piston taken between two successive exposures, as proposed by Colavita (1999b) for the PTI interferometer and successfully applied by Malbet et al. (1998). However, jitter analysis (as illustrated in Sect. 5.2) cannot be tested and validated as long as the extra-sources of vibrations due to VLTI instabilities (delay lines, adaptive optics, etc.), hardly calibratable, are clearly identified and suppressed. Note as well that the use of the accurate fringe tracker FINITO (Gai et al. 2002), soon expected to operate on the VLTI, should drastically reduce the jitter attenuation, hence allowing integration on much longer times than the coherence time of the atmosphere in order to reach fainter stars.

\section{The amdlib data reduction software}

A dedicated software to reduce AMBER observations has been developed by the AMBER consortium. This consists of a library of C functions, called amdlib, plus high-level interface programs. The amdlib functions are used at all stages of AMBER data acquisition and reduction: in the observation software (OS) for wavelength calibration and fringe acquisition, in the (quasi) real time display program used during the observations, in the online data reduction pipeline customary for ESO instruments, and in various offline front end applications, noticeably a Yorick implementation (AmmYorick). The amdlib library is meant to incorporate all the expertise on AMBER data reduction and calibration acquired throughout the life of the instrument, which are bound to evolve with time.

The data obtained with AMBER ("raw data") consist of an exposure, i.e., a time series of frames read on the infrared camera, plus all relevant information from AMBER sensors, observed object, VLTI setup, etc., stored in FITS TABLE format, according to ESO interface document VLT-ICD-ESO-150001826. Saving the raw, uncalibrated data, although more spaceconsuming, permits us to benefit afterwards, by replaying the calibration sequences and the data reduction anew, from all the improvements that could have been deposited in amdlib in the meantime.

The library contains a set of "software filters" that refine the raw data sets to obtain calibrated "science data frames". This treatment is performed on all of the raw data frames, irrespective of their future use (calibration or observation). A second set of functions performs high-level data extraction on these calibrated frames, either to compute the V2PM (see Sect. 4.3) from a set of calibration data or to extract the visibilities from a set of science target observations, the end product in this case being a reduced set of visibilities per object, stored in the optical interferometry standard OI_FITS format (Pauls et al. 2005).

\subsection{Detector calibration}

First, all frames pixels are tagged valid if not present in the currently available bad pixel list of the AMBER detector. Then they are converted to photoevent counts. This step necessitates, for each frame, precisely modelling of the spatially and temporarily variable bias added by the electronics. The detector exhibits a pixel-to-pixel (high frequency) bias whose pattern is constant in time but which depends on the detector integration time (DIT) and the size and location of the subwindows read on the detector. Thus, after each change in the detector setup, a new pixel bias map (PBM) is measured prior to the observations by averaging a large number of frames acquired with the detector facing a cold shutter ${ }^{2}$. This PBM is then simply removed from all frames prior to any other treatment.

Once this fixed pattern has been removed, the detector may still be affected by a time-variable "line" bias, i.e., a variable

\footnotetext{
2 Due to mechanical overheads, "hot dark" observations, i.e., using only an ambient temperature beam shutter external to the dewar of the detector, are currently used to compute the PBM.
} 
offset for each detector line. This bias is estimated for each scan line and each frame as the mean value of the corresponding line of masked pixels ("DK" column in Fig. 1) and then substracted from the rest of the line of pixels. The detector has an image persistence of $\sim 10 \%$; consequently, all frames are corrected for this effect before calibration. Pixels are then converted to photoevent counts by multiplying by the pixel's gain. Currently the map of the pixel gains used is simply a constant $\mathrm{e}^{-} / \mathrm{ADU}$ value (see Table 1) multiplied by a "flat field" map acquired during laboratory tests. Finally, the rms of the values in the masked pixel set, which were calibrated as the rest of the detector, gives the frame's detector noise.

\subsection{Image alignment and science data production}

Once the cosmetics on the pixels is done, amdlib corrects the data from the spatial distortions present in the image. Presently, the only corrected effect is a displacement of the spectra acquired in the "photometric channels" (labeled P1, P2, P3 in Fig. 1) with regards to the fringed spectrum in the interferometric channel. This displacement of a few pixels in the spectral dispersion direction is due to a slight misalignment of the beam-splitters described in Sect. 2.1, and correcting for this effect is mandatory for computing the DC continuum interferogram (Eq. (11)). The calibration of this displacement is performed by amdlib during the spectral calibration procedure, one of the first calibration sequences to be performed prior to observations.

Finally, each frame is converted to the handier "science data" structure, which contains only the calibrated image of the "interferometric channel" and (up to) three 1D vectors, the corresponding instantaneous photometry of each beam, corrected for the above-mentioned spectral displacement.

\subsection{Calibration matrix computation}

Computation of the V2PM matrix is performed by the function amdlibComputeP $2 \mathrm{vm}$ (). This function processes the 4 or 9 files described in Sect. 3.2 applying by each of them the detector calibration, image alignment, and conversion to "science data" described above, then computing the $v_{k}^{i}$ (Eq. (10)) and the carrying waves $c_{k}^{i j}$ and $d_{k}^{i j}$ of the V2PM matrix (Eq. (13)). The result is stored in a FITS file, improperly called, for historical reasons, "the P2VM"3.

The P2VM matrix is the most important set of calibration values needed to retrieve visibilities. The shape of the carrying waves (the $c_{k} \mathrm{~s}$ and $d_{k} \mathrm{~s}$ ) and, in lesser measure, the associated $v_{k} \mathrm{~s}$ are the imprints of all the changes in intensity and phase that the beams suffer between the output of each fiber and detection on the infrared camera. Any change in the AMBER optics situated in this zone, either by moving, e.g., a grating, or just thermal long-term effects, render the P2VM unusable. Thus, the P2VM matrix must be recalibrated each time a new spectral setup is called that involves changing the optical path behind the fibers.

All the instrument observing strategies and operations are governed by the need to avoid unnecessary optical changes, and care is taken at the operating system level to assure a recalibration of the P2VM whenever a "critical" motor affecting the optical path is set in action. To satisfy these needs, the P2VM computation has been made mandatory prior to science observations and is given an unique ID number. All the science data files produced after the P2VM file inherit this ID, which associates

\footnotetext{
3 Actually "the V2PM" should be the proper name.
}

them with their "governing" calibration matrix. The amdlib library takes the opportunity of the P2VM file being pivotal to the data reduction, and unique, to make it a placeholder for all the other calibration tables needed to reduce the science data, namely the spectral calibration, bad pixels, and flat field tables.

\subsection{From science data to visibilities}

The computation of visibilities is performed by the amdlibExtractVisibilities() function, using a valid P2VM file. Then amdlibExtractVisibilities () is able to perform visibility estimates on a frame-by-frame basis, or over a group of frames, called bin.

The amdlibExtractVisibilities() function, in sequence is

1. invert the V2PM calibration matrix;

2. extract raw visibilities;

3 . correct for biases, compute debiased $V^{2}$ visibilities;

4. compute phase closures;

5. compute cross spectra;

6. fit piston values from cross spectra;

7. write the OI-FITS output file.

A typical data reduction process will first process all raw data files related to the calibration procedures performed before acquiring the science data, thus performing spectral calibration (e.g., using the command line program amdlibComputeSpectralCalibration), then P2VM file computation (e.g., using the command line program amdlibComputeP2vm). Once the P2VM file is computed, it contains all the calibration quantities needed to process science object observations. One then uses the amdlibExtractVis program on a science data set to get the final OI-FITS file containing the measured science object visibilities.

\section{Illustrations and discussions}

This section aims to present, step-by-step, the data reduction procedures performed on real interferometric measurements arising from VLTI observations. Results are discussed, focusing on key points in the process.

\subsection{Fringe fitting}

Assuming the calibration process has been properly performed following Sect. 3.2, the first step in the derivation of the observables is to estimate the real and imaginary parts of the coherent flux. This is done by inverting the calibration matrix and obtaining the P2VM matrix, shown by Eqs. (17) and (18). Figure 4 gives an example of the fringe fitting process for an observation of the calibrator star HD135382 with three telescopes.

However, before going further in the data reduction process, it might be worthwhile for the users to check the validity of the fit and then to detect any potential problems in the data. Such a step can be easily done by computing the residual $\chi_{\text {res }}^{2}$ between the measurements $m_{k}$ and the model $\widetilde{m}_{k}$ :

$\left[\widetilde{m}_{k}\right]=\operatorname{V} 2 \mathrm{PM}\left[\widetilde{R}^{i j}, \widetilde{I}^{i j}\right]$

and

$\chi_{\text {res }}^{2}=\left[\widetilde{m}_{k}-m_{k}\right]^{\mathrm{T}} C_{\mathrm{M}}^{-1}\left[\tilde{m}_{k}-m_{k}\right]$. 


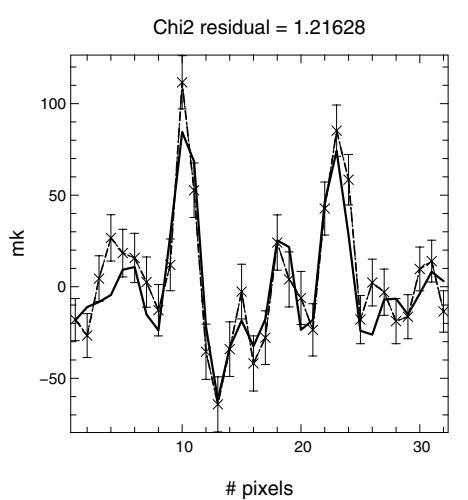

Fig. 4. Example of fringe-fitting by the carrying waves in the 3-telescope case. The DC-corrected interferogram is plotted (dashdot line) with the error bars. The result of the fit is overplotted (solid line).

Using this checking procedure, the user can verify two critical points of the data processing:

- the correct subtraction of the DC component (see Eq. (11)): if such a condition is not fulfilled, the computed visibility will inevitably be biased since the fringe fitting by the carrying waves supposes the only presence of specific frequencies, that is, the spatial coding frequencies of the instrument. A wrong DC subtraction might occur with sudden atmospheric changes between the recording of the interferometric channel and the associated photometric ones, as these channels are not on the same line of the detector, as mentioned in Sect. 4.2.

- the use of a correct bad pixel map: if not, the presence of bad pixels induces high frequencies in the fringes, which cannot be taken into account by the carrying waves, driving one to compute biased visibility, as well. Note that the bad pixel map is computed every time a detector calibration is performed in the maintenance procedure.

\subsection{Fringe criterion and fringe selection}

For each frame of the set of data, Eq. (20) provides an estimation of the fringe signal-to-noise ratio. As an example, Fig. 5 presents 100 fringes recorded on the detector during the 2-telescope observation of the calibrator $\epsilon$ Sco in July 2005, first in the order they appeared during the observation and then after re-ordering them following the fringe criterion.

The aim of computing this criterion can be twofold: (i) during the observations, as mentioned in Sect. 3.4, it allows us to detect the fringes and therefore to initiate the recording of the data only when it is meaningful; and (ii) calculated a posteriori during the data reduction phase, it enables us to select the best frames (in terms of SNR) before estimating the observables. This second point is especially important where frames are recorded in the presence of strong and variable fringe jitter.

In the ideal and unrealistic case where the fringes are not moving during the integration time, the fringe contrast is not attenuated by vibrations, and the frame-by-frame estimated visibility is constant, no matter what the photometric flux level is in each arm of the interferometer. As a result, the visibility as a function the function of the fringe SNR is constant, with the error bars increasing as the fringe SNR decreases. This is illustrated in Fig. 6 (left). To obtain this set of jitter-free data, we have built interferograms using the carrying waves of the calibration matrix that simulate perfectly stable AMBER fringes.
Then, we have added the photometry taken on the $\epsilon$ Sco data, which allowed us to keep realistic photometric realizations taking the correct transmissions of the instrument into account. In that case, selecting the best fringes has no other goal than to improve the SNR of the observables by excluding the data with poor flux.

In the presence of atmospheric turbulence and lack of a fringe tracker, the fringes are moving during the integration time, leading to lower the visibility. On average, the squared visibility is attenuated by a factor $\exp \left(-\sigma_{\phi_{h f}^{p}}^{2}\right)$, where $\sigma_{\phi_{h f}^{p}}^{2}$ is the variance of the atmospheric high pass jitter, as explained in Sect. 3.6.2. The frame-by-frame visibility, though, undergoes a random attenuation around this average loss of contrast. An example of the effect of the atmospheric jitter is given in Fig. 6 (middle), where a previous set of simulated data has been used, adding a frameby-frame random attenuation taking the $\tau=25 \mathrm{~ms}$ integration time of the $\epsilon$ Sco observation into account. Once again, fringe selection only enables us to increase the SNR of the observables here.

However, when we look at the real set of data obtained from the observation of $\epsilon$ Sco, we obtain the plot displayed in Fig. 6 (right). The dispersion of the visibility, especially for low fringe SNR is unexpectedly large and can definitively not be explained by pure atmospheric OPD vibrations. As a matter of fact, these variations are due to the present strong vibrations along the VLTI instrumentation (adaptive optics, delay lines, etc.), as this effect was previously revealed by the VINCI recombiner. These vibrations strongly reduce the fringe contrast and subsequently the value of the estimated visibilities, which explains the behavior of the visibilities as a function of the fringe SNR. Indeed, when the visibility tends toward 0 , because of severe jitter attenuation, the fringe criterion tends toward 0 as well. In contrast, the visibility plotted as a function of the fringe SNR saturates for high values of the latter.

The major issue is that such an effect is hardly calibratable because potentially non stationary. Hence, one convenient way to overcome the problem, beside increasing the error bars artificially to take this phenomenon into account, is to only select the fringes that are less affected by the vibrations, that is, the fringes with the highest fringe SNR. One can then choose the percentage of selected frames from which the visibility will be estimated. The threshold must be chosen according to the following tradeoff: reducing the number of frames considered allows to get rid of most of the jitter attenuation, but, from a certain number when the sample is not large enough to perform statistics, it increases the noise on the visibility. Furthermore, it leads to mis-estimate the quadratic bias (see Eq. (25)), which is by essence a statistical quantity, and consequently drives to introduce a bias in the visibility.

Obviously, such a selection process must be handled with care, and its robustness with regard to the selection level has to be established for any given observation. In other words, for this method to be valid, the expected value for the calibrated visibility must remain the same, with only the error bars changing and eventually reaching a minimum at some specific selection level. In particular, this method seems well adapted, above all, to cases where the calibrator exhibits a magnitude close to the source's one, where the visibility distribution versus the SNR is expected to behave similarly. Going into further details af this point is nevertheless beyond the scope of this paper as it will be deeply developed in Millour et al. (2007). However note that we experimentally found this procedure to be generally robust, and for typical observations performed until now with the VLTI, 

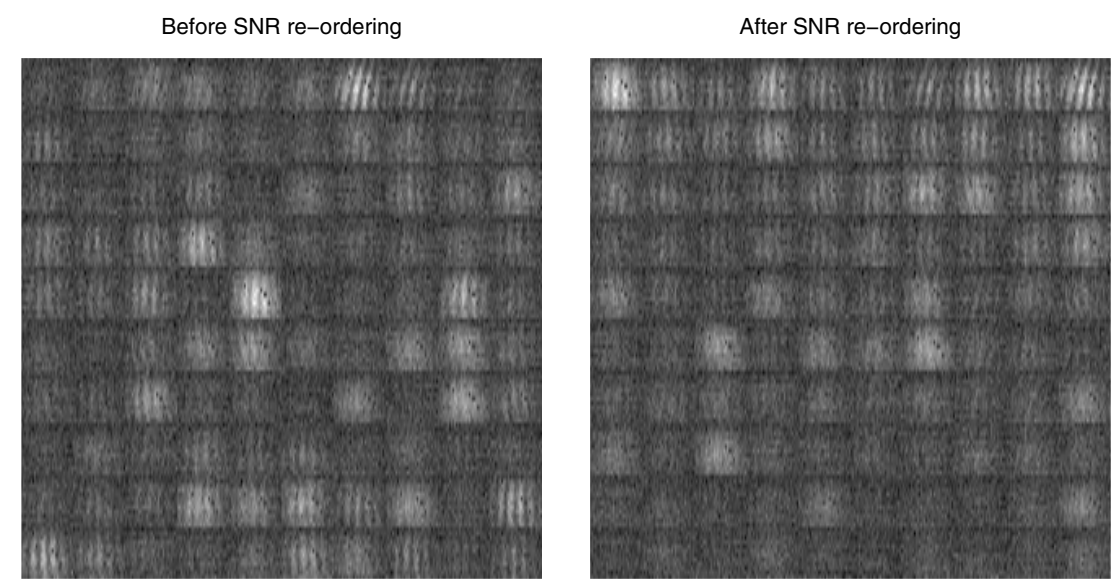

Fig. 5. Left: sample of 100 successive interferograms as recorded during the observation with two telescopes of $\epsilon$ Sco in the low spectral-resolution mode. Right: Re-ordering of this sample using the fringe SNR criterion (from left to right, bottom to top). Note that some frames that are on the bottom of the right panel (that is, with relatively low SNR) appear to be brighter than some above them (that is, the flux is higher). However these frames do not exhibit fringes, which explains their positions.
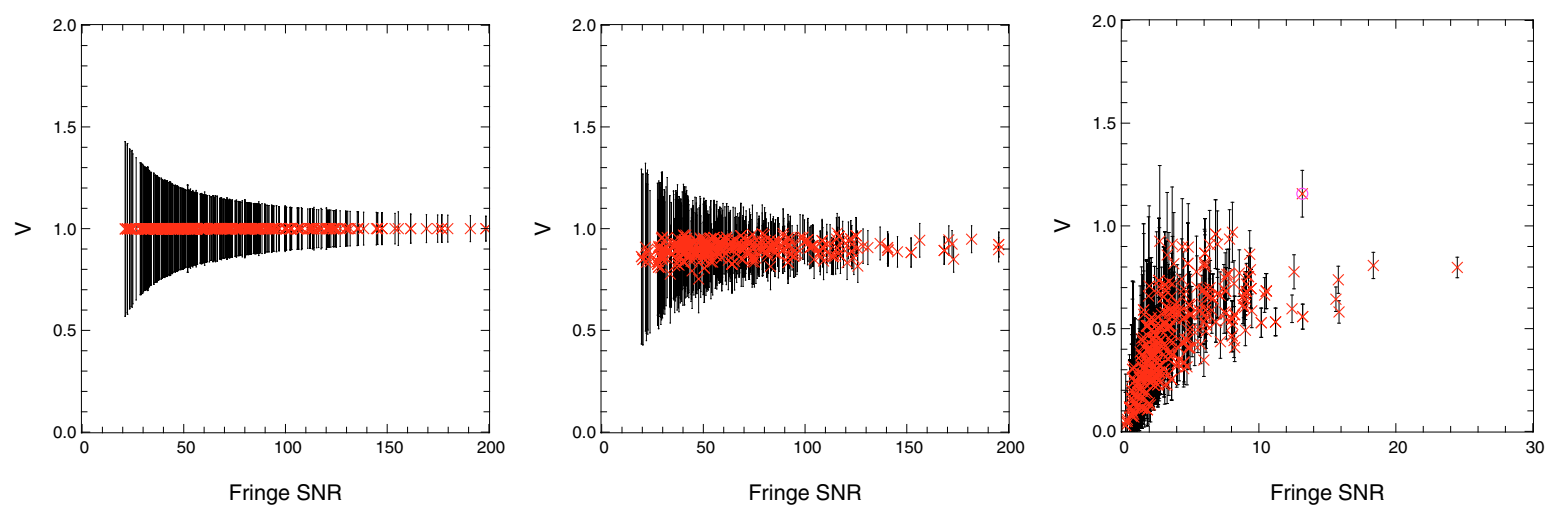

Fig. 6. Visibility as a function of the fringe SNR criterion. Left: for jitter-free simulated data, using the real photometry observed on $\epsilon$ Sco. The fringe contrast was set to 1. Middle: same as the previous one, but atmospheric jitter attenuation has been added, corresponding to an integration time of $\tau=25 \mathrm{~ms}$. Right: real $\epsilon$ Sco observation. The encircled data point on the plot, well above the other ones, is typical of a bad fit of the associated fringe, as explained in Sect. 5.1. Note that in the first two cases, the maximum of the fringe SNR is higher than in the real case. Indeed, in the simulated data, the noise on the coherent flux only arises from the photometry $P^{i}$. In the real case, however it also depends on the noise on the interferograms $i_{k}$ (see Eq. (19)).

choosing $20 \%$ of the frames as the final sample appeared to be a good compromise.

Note that, in order to produce the curve of Fig. 6, visibilities were computed frame by frame (i.e. $M=1$ ). Thus, the semiempirical calculation of the error bars given below in Sect. 5.3 does not work, and one has to use a full theoretical expression of the noise. From an analysis in Fourier space, Petrov et al. (2003) show that the theoretical error on the frame-by-frame visibility could be written:

$\sigma^{2}\left(V^{i j}\right)=\frac{n^{i}+n^{j}+N_{\mathrm{pix}} \sigma^{2}}{n^{i} n^{j}}$

where $n^{i}(t)=\sum_{k}^{N_{\text {pix }}} v_{k}^{i} P^{i}(t)$ is the total flux in the $i$ th beam. This computation is not fully adapted to the AMBER data processing using 3 telescopes, since in that case the Fourier peaks are overlapping. Nevertheless, it gives a rough estimation of the noise level, within a factor of 2 , which is sufficient for the analysis discussed here.

Finally, despite fringe selection has been performed to deal at best with the uncalibratable VLTI vibrations, the dispersion of the selected visibilities still has to be quadratically added to the error bar arising from the fundamental noises (as computed in
Sect. 5.3), in order to account for the reminiscent jitter attenuation, which has been reduced but not totally canceled out.

\subsection{Visibilities and associated errors}

The raw squared visibility (that is biased by the atmosphere) and its associated error bar were estimated from the ensemble average of $M$ exposures, using Eqs. (24) and (27), respectively. Figure 7 gives an example of the computed squared visibility in the low resolution mode, arising from the observation of the calibrator $\epsilon$ Sco. For the example considered above, we find $V^{2}=0.2721 \pm 0.0152$ after averaging the spectrally dispersed visibilities.

In order to validate the computation of the error bars, we used bootstrapping techniques (Efron \& Tibshirani 1993). By making sampling with replacement, such a method constructs a large population of $N$ elements ( $N$ estimated squared visibility) from the original measurements ( $M$ coherent and photometric fluxes). If $N$ is large enough, the statistical parameters, i.e. the mean value and the dispersion of this population are converging toward the expected value and the root mean square of the estimated parameters, respectively. $N$ large enough, these quantities can be calculated by fitting a Gaussian distribution $p\left(V^{2}\right)$ 


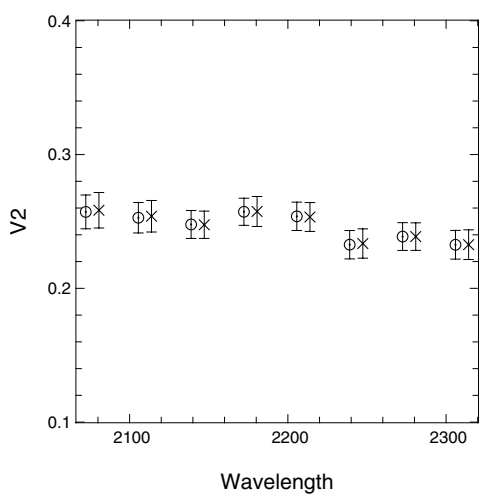

Fig. 7. Estimation of the raw squared visibility and its error-bars as a function of the wavelength for the observed calibrator $\epsilon$ Sco in low resolution mode. Visibility crosses and corresponding errors bars are computed thanks to Eqs. (24) and (27), respectively. Circles and corresponding errors bars arise from the bootstrapping technique. For the sake of clarity, visibilities have been slightly shifted to the right and to the left of the corresponding wavelengths.

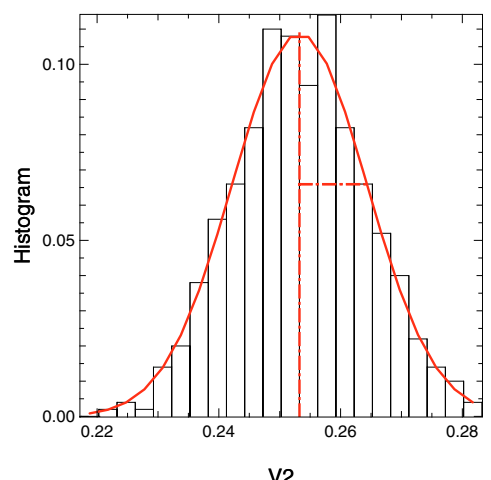

Fig. 8. Histogram of the bootstrapped population of estimated squared visibilities for a given wavelength. The fit of this histogram by a Gaussian function is superimposed. The mean value and the root mean square of the Gaussian distribution give the statistics of the estimated visibility.

to the histogram of the bootstrapped population. Figure 8 gives an example of the histogram and the resulting Gaussian fit. Using this method with $N=500$, we find for the same set of data $V^{2}=0.2719 \pm 0.0149$, which is in excellent agreement with previous computation.

Note that, although we observed this object in the low resolution mode with reasonably high flux, we find a relative error on the order of $6 \%$. Such a large error bar is due to the atmospheric and intrumental jitter that, in the absence of fringe tracking, prevents an integration time from being longer than a few tenth of milliseconds. When this latter device will be available, we expect to lower this error below the $1 \%$ level, down to $0.01 \%$ for the brightest cases (assuming perfect fringe tracking, see Malbet et al. 1998; Petrov et al. 2007). But it is not possible to achieve AMBER's ultimate performances at that time.

\subsection{Notion of instrumental contrast in AMBER}

Given the calibration of the instrument described in Sect. 3.2 and its subsequent use for the estimation of the visibility in Sect. 3.5.1, the instrumental contrast of AMBER is self calibrated. In other words, the response of the AMBER/VLTI instrument to the observation of a point source - in the absence of atmospheric turbulence - does not depend on the

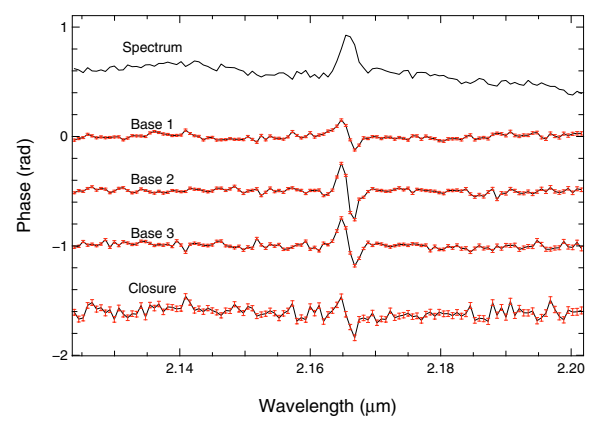

Fig. 9. Example of differential phases and closure phase computation on an observed object with a rotating feature in the $\mathrm{Br} \gamma$ emission line ( $\alpha$ Arae, see Meilland et al. (2007) for a complete description and interpretation of these phases).

instrumental contrast but only on the visibility of the internal source (see Eq. (24)). Thus, if one wants to characterize the instrumental contrast, that is, the total loss of contrast due to the instrumentation, one needs to use another estimator in which the calibration part (the use of the knowledge of the instrument characteristics) is skipped. We thus can use the classical definition of contrast in the image plane, directly measured "by eye" from the interferograms $i_{k}$, recorded by pair of telescopes (as for the computation of the carrying waves). In spatial coding, this can be done for each pixel of the interferogram. Using Eqs. (3) and (10), we get:

$C_{r}^{i j}=C_{\mathrm{B}}^{i j}\left(\frac{1}{N_{\mathrm{pix}}} \sum_{k} \frac{2 \sqrt{P^{i} v_{k}^{i} P^{j} v_{k}^{j}}}{P^{i} v_{k}^{i}+P^{j} v_{k}^{j}}\right)$.

Such an equation says that the instrumental contrast loss depends on two separate effects: (i) the polarization mismatch between the beams after the polarizers (vectorial effect) and (ii) the misalignment of the interfering beams (taken into account in the product $v_{k}^{i} v_{k}^{j}$ ), together with the photometric inbalance between the two beams (scalar effect). Both effects are compensated when computing the visibility from the P2VM.

\subsection{Closure phase}

In the current situation, closure phases are computed using the estimator of Eq. (28), but a previous frame selection is performed before making the ensemble average of the bispectrum, because in all the data available there was a very low amount of frames that simultaneously presented three fringe patterns. We chose an empirical selection criterion as the product of the three individual fringe SNR criteria (as defined by Eq. (20)). Internal error bars the closure phases are computed statistically, taking the root mean square of all the individual frames divided by the square root of the number of frames (assuming statistical independence of the frames), since the estimations of the tested theoretical error bars do not give satisfactory results up to now.

An example of closure phase and closure phase error bars is given in Fig. 9. The object is $\alpha$ Arae, which contains a rotating feature in the Br $\gamma$ emission line (Meilland et al. 2007). A full description of how the closure phase and closure phase errors are computed will be part of the second paper on the AMBER data reduction (Millour et al. 2007). 

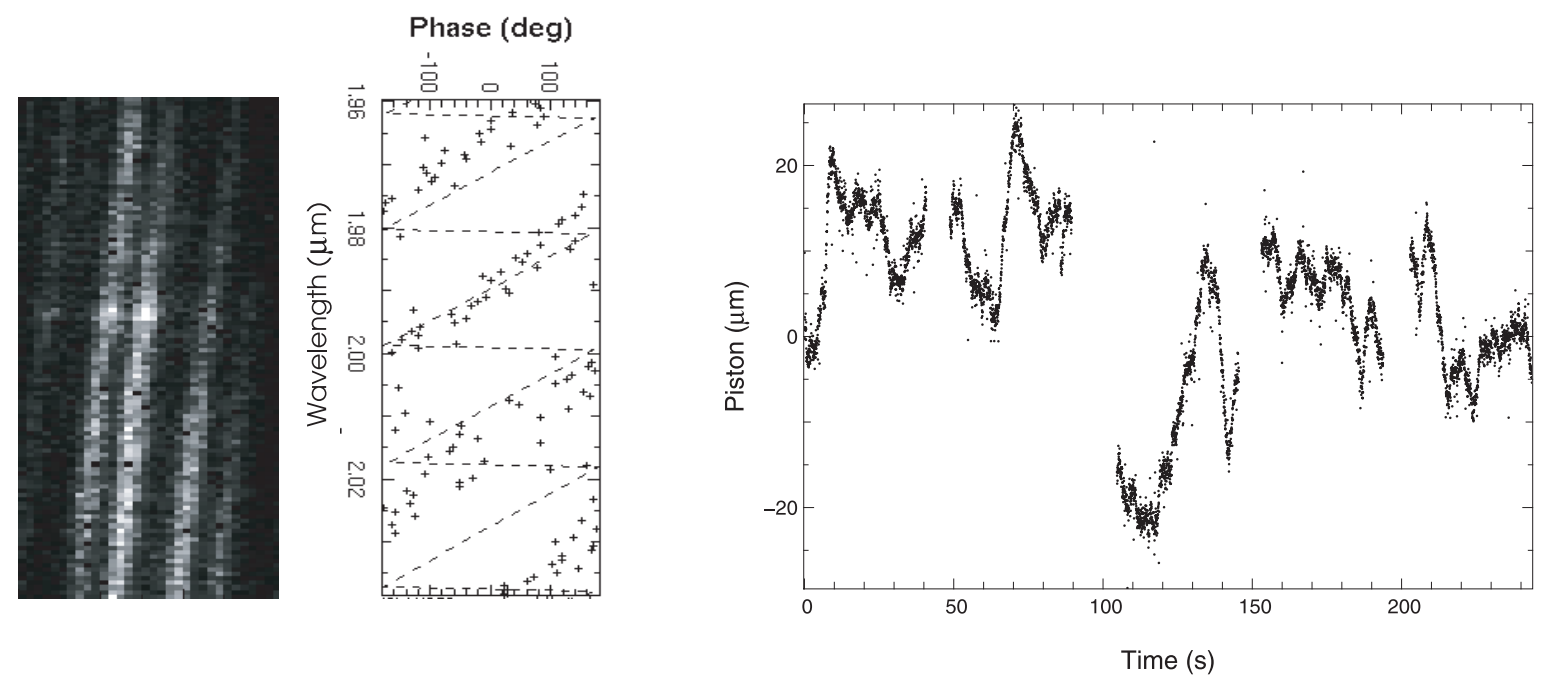

Fig. 10. Piston estimation from the fringe pattern. From left to right is (i) the raw fringe pattern, the corresponding phase; (ii) the estimated linear component of the phase from the least square fit; and (iii) a piston time-sequence over $250 \mathrm{~s}$. Note that the piston rms is around $15 \mu \mathrm{m}$, which agrees with the average atmospheric conditions recorded in Paranal (Martin et al. 2000).

\subsection{Differential phase and piston}

An example of differential phases is given in Fig. 9. It is computed from the ensemble average of the cross spectrum as defined in the estimator of Eq. (30). Frame-by-frame correction of its linear part (i.e. unwrapping) has been performed. The resulting differential phase shows a typical rotation signal that is fully described in Meilland et al. (2007). Currently, like the closure phase, the internal error bars are computed statistically assuming that the differential phases are statistically independent frame to frame. An extensive description of the data processing and of the informations that can bring the differential phases will be described in our second paper (Millour et al. 2007).

The computation of the linear component of the differential phase; that is, the piston estimation is done on each spectral band separately $(J, H$, or $K)$, using the least-square method described in Sect. 3.5.4. This algorithm was extensively tested on the sky and validated as a part of the observing software of the AMBER instrument. An example of the fitting process, as well as of the piston estimate is given in Fig. 10.

\section{Conclusions}

We have described the data reduction formalism of the AMBER/VLTI instrument, that is, the principles of the algorithm that lead to the computation of the AMBER observables. This innovative signal processing is performed in three main steps: (i) the calibration of the instrument, which provides the calibration matrix that gives the linear relationship between the interferogram and the complex visibility; (ii) the inversion of the calibration matrix to obtain the so-called P2VM matrix and then the complex visibility; and (iii) the estimation of the AMBER observables from the complex visibility, namely the squared visibility, the closure phase, and the differential phase.

Note that this analysis requires the calibration matrix to be both perfectly stable in time and very precise, i.e. recorded with a much higher SNR than the SNR of the interferograms. If the instrument is not stable between the calibration procedures and the observations, the P2VM will drift and, as a result, the estimated observables will be biased. And if the calibration is not precise enough, it will be the limiting factor for the SNR of the observables. For the latter problem, it is thus recommended to set, during the calibration process, an integration time that insures a P2VM accuracy at least a factor of 10 higher than the accuracy expected on the measurements. To check the former problem of stability, it is advised to record one P2VM before and one P2VM directly after the observation. This procedure allows us to quantify the drift of the instrument along the observations and to potentially reject the data if the drift appears to be significant. Note, however, that stability measurements in laboratory have shown the AMBER instrument to be generally stable on the hour scale at least.

Regarding the closure phase and the differential phase, we have produced the theoretical estimators arising from the specific technique of AMBER data reduction, as well as brief illustrations from real observations. A thorough analysis, including practical issues and performances, of these two observables, which deal the phase of the complex visibility, will be given in a forthcoming paper (Millour et al. 2007)

For the squared visibility, we have defined an estimator that is self-calibrated from the instrumental contrast and we have investigated its biases. The quadratic bias, which is an additive quantity and results in the quadratic estimation in the presence of zero-mean value additive noise, can be easily corrected, providing the computation of the error of the fringe measurements. Atmospheric and instrumental biases, which attenuate the visibility through multiplicative attenuation factors, come from (i) the high frequency fringe motion during the integration time - namely the jitter - and (ii) from the loss of spectral coherence when the fringes are not centered at the zero optical path difference - namely the atmospheric differential piston. The latter can be estimated from the differential phase and its consecutive attenuation can be corrected by knowing the shape of the spectral filter and the resolution of the spectrograph. When strictly arising from atmospheric turbulence, The former can be calibrated by a reference source, provided it has been observed shortly before/after the object of interest. When instrumental, hardly calibratable vibrations accumulate in the jitter phenomenon, as presently the case for the VLTI, we propose a method based on sample selection that allows reduction of the attenuation and the associated dispersion on the visibilities.

However at this point, because of the presence of these instrumental vibrations and because of the absence of the FINITO 
fringe tracker, it is possible neither to develop an optimized tool for identifying and calibrating the biases coming from the atmospheric turbulence nor to present an analysis of the ultimate performances of the AMBER/VLTI instrument. These points will be developed in our next paper on the AMBER data reduction methods, once the problems mentioned above, which are independent of the AMBER instrument, would have been resolved.

Acknowledgements. The AMBER project ${ }^{4}$ was founded by the French Centre National de la Recherche Scientifique (CNRS), the Max Planck Institute für Radioastronomie (MPIfR) in Bonn, the Osservatorio Astrofisico di Arcetri (OAA) in Firenze, the French Region "Provence Alpes Côte D'Azur" and the European Southern Observatory (ESO). The CNRS funding has been made through the Institut National des Sciences de l'Univers (INSU) and its Programmes Nationaux (ASHRA, PNPS, PNP).

The OAA co-authors acknowledge partial support from MIUR grants to the Arcetri Observatory: A LBT interferometric arm, and analysis of VLTI interferometric data and From Stars to Planets: accretion, disk evolution and planet formation and from INAF grants to the Arcetri Observatory Stellar and Extragalactic Astrophysics with Optical Interferometry. C. Gil work was supported in part by the Fundação para a Ciência e a Tecnologia through project POCTI/CTE-AST/55691/2004 from POCTI, with funds from the European program FEDER.

The preparation and interpretation of AMBER observations benefit from the tools developed by the Jean-Marie Mariotti Center for optical interferometry $\mathrm{JMMC}^{5}$ and from the databases of the Centre de Données Stellaires (CDS) and of the Smithsonian/NASA Astrophysics Data System (ADS).

The data reduction software amdlib is freely available on the AMBER site http://amber.obs.ujf-grenoble.fr. It has been linked to the public domain software Yorick ${ }^{6}$ to provide the user-friendly interface ammyorick.

\section{References}

Colavita, M. M. 1999a, BAAS, 31, 1407

Colavita, M. M. 1999b, PASP, 111, 111

Connes, P., Shaklan, S., \& Roddier, F. 1987, Interferometric Imaging in Astronomy, 165

Coudé Du Foresto, V., Ridgway, S., \& Mariotti, J.-M. 1997, A\&AS, 121, 379

Coudé du Foresto, V., Faucherre, M., Hubin, N., \& Gitton, P. 2000, A\&AS, 145, 305

Dyer, S. D., \& Christensen, D. A. 1999, Opt. Soc. Am. J. A, 16, 2275

Efron, B., \& Tibshirani, R. J. 1993, An Introduction to the Bootstrap. Monographs on Statistics and Applied Probability, 57 (New York: Chapman \& Hall)

Gai, M., et al. 2002, Scientific Drivers for ESO Future VLT/VLTI Instrumentation Proceedings of the ESO Workshop held in Garching, Germany, 11-15 June, 2001, 328

Guilloteau, S. 2001, Millimetre Interferometers, in IRAM Milimeter Interferometry Summer School, 2, 15

Guyon, O. 2002, A\&A, 387, 366

Kervella, P., Gitton, P. B., Segransan, D., et al. 2003, Proc. SPIE, 4838, 858

Kervella, P., Ségransan, D., \& Coudé du Foresto, V. 2004, A\&A, 425, 1161

Kirkpatrick, S., Gelatt, C. D., \& Vecchi, M. P. 1983, Science, 220, 671

Longueteau, E., Delage, L., \& Reynaud, F. 2002, Appl. Opt., 41, 5835

LeBouquin, J. B., Rousselet-Perraut, K., Kern, P., et al. 2004, A\&A, 424, 719
Malbet, F., Berger, J.-P., Colavita, M. M., et al. 1998, ApJL, 507, L149

Malbet, F., et al. 2001, AMBER Intrument Analysis Report, VLT-SPE-AMB-15830-0001

Martin, F., Conan, R., Tokovinin, A., Stee, P., Vannier, M., et al. 2000, A\&AS, 144, 39

Mège, P., Malbet, F., \& Chelli, A. 2003, Proc. SPIE, 4838, 329

Meilland, A., Stee, P., Vannier, M., et al. 2007, A\&A, 464, 59

Millour, F., et al. 2007, in preparation

Mourard, D., Bonneau, D., Glentzlin, A., et al. 2000, Proc. SPIE, 4006, 434

Mourard, D., Tallon-Bosc, I., Rigal, F., et al. 1994, A\&A, 288, 675

Pauls, T. A., Young, J. S., Cotton, W. D., \& Monnier, J. D. 2005, PASA, in press

Papoulis, A. 1984, Probability, random, variables and stockastic processes (New York: McGraw-Hill), 2nd ed.

Perrin, G. 2003, A\&A, 398, 385

Petrov, R. G., Vannier, M., Lopez, B., et al. 2003, EAS Publications Series, 8, 297

Petrov, R. G., Malbet, F., Weigelt, G., et al. 2007, A\&A, 464, 1

Roddier, F. 1986, Opt. Comm., 60, 145

Roddier, F., \& Lena, P. 1984, J. Opt., 15, 171

Ruilier, C., Conan, J.-M., \& Rousset, G. 1997, Integrated Optics for Astronomical Interferometry, 261

Shaklan, S., \& Roddier, F. 1988, Appl. Opt., 27, 2334

Tatulli, E., Mège, P., \& Chelli, A. 2004, A\&A, 418, 1179

1 Laboratoire d'Astrophysique de Grenoble, UMR 5571 Université Joseph Fourier/CNRS, BP 53, 38041 Grenoble Cedex 9, France

2 INAF-Osservatorio Astrofisico di Arcetri, Istituto Nazionale di Astrofisica, Largo E. Fermi 5, 50125 Firenze, Italy

3 Laboratoire Universitaire d'Astrophysique de Nice, UMR 6525 Université de Nice - Sophia Antipolis/CNRS, Parc Valrose, 06108 Nice Cedex 2, France

4 Max-Planck-Institut für Radioastronomie, Auf dem Hügel 69, 53121 Bonn, Germany

5 Laboratoire Gemini, UMR 6203 Observatoire de la Côte d'Azur/CNRS, BP 4229, 06304 Nice Cedex 4, France

6 European Southern Observatory, Casilla 19001, Santiago 19, Chile

7 ONERA/DOTA, 29 av de la Division Leclerc, BP 72, 92322 Chatillon Cedex, France

8 Centre de Recherche Astronomique de Lyon, UMR 5574 Université Claude Bernard/CNRS, 9 avenue Charles André, 69561 Saint Genis Laval Cedex, France

9 Division Technique INSU/CNRS UPS 855, 1 place Aristide Briand, 92195 Meudon Cedex, France

10 IRCOM, UMR 6615 Université de Limoges/CNRS, 123 avenue Albert Thomas, 87060 Limoges Cedex, France

11 European Southern Observatory, Karl Schwarzschild Strasse 2, 85748 Garching, Germany

12 Kiepenheuer Institut für Sonnenphysik, Schöneckstr. 6, 79104 Freiburg, Germany

13 Departamento de Astronomia, Universidad de Chile, Chile

14 Instituut voor Sterrenkunde, KU-Leuven, Celestijnenlaan 200D, 3001 Leuven, Belgium

15 Centro de Astrofísica da Universidade do Porto, Rua das Estrelas, 4150-762 Porto, Portugal

16 Laboratoire Astrophysique de Toulouse, UMR 5572 Université Paul Sabatier/CNRS, BP 826, 65008 Tarbes Cedex, France

\footnotetext{
${ }^{4}$ The structure and members of the AMBER Consortium can be found in the website: http://amber.obs.ujf-grenoble.fr

5 The JMMC is a center providing software tools for optical interferometry described at the website: http://www.jmmc . fr

${ }^{6}$ http://yorick. sourceforge.net
} 